\title{
Microfluidic Live-Imaging Technology to Perform Research Activities in 3D Models
}

\section{Capuzzo Arnaud Martino* and Daniele Vigo}

Department Veterinary Medicine, Università di Milano, USA

*Corresponding author: Capuzzo Arnaud Martino, Department Veterinary Medicine, Università di Milano, Via Lodi 626900 Lodi, USA

Research Article

Volume 4 Issue 1

Received Date: April 14, 2021

Published Date: May 10, 2021

DOI: $10.23880 /$ aabsc- 16000160

\section{Abstract}

Morphological dissimilarity and its evolution over time are one of the most unexpected variations found when comparing cell cultures in 2D and 3D. Monolayer cells appear to flatten in the lower part of the plate, adhering to and spreading in the horizontal plane while not extending vertically. Consequently, cells developed in two dimensions have a forced apex-basal polarity. Co-cultivation and crosstalking between multiple cell types, which control development and formation in the in vivo counterpart, are possible in 3D cultures. With or without a scaffold matrix, 3D model culture may exhibit more in vivo-like morphology and physiology. 3D cultures mimic relevant physiological cellular processes, transforming them into one-of-akind drug screening platforms. The structures and dynamics of regulatory networks, which are increasingly studied with live-imaging microscopy, must be considered to help and guarantee the functional maintenance of a 3D structure. However, commercially available technologies that can be used for current laboratory needs are minimal, despite the need to make it easier to acquire cellular kinetics with high spatial and temporal resolution, in order to improve visual efficiency and, as a result, experimentation performance. The CELLviewer is a newly developed multi-technology instrument that integrates and synchronizes the work of various scientific disciplines. The aim of this study is to test the device using two different models: a single Jurkat cell and an MCF-7 1 spheroid. The two models are loaded into the microfluidic cartridge for each experiment after they have been grown and captured in time-lapse for a total of 4 hours. The samples used are tracked under the operation of the optics after adaptive autofocus, while slipping inside the cartridge chamber and the 3D rotation was successfully obtained experimentally. The MitoGreen dye, a fluorescence marker selectively permeable to live cells, was then used to determine cell viability. To measure the model diameter, construct fluorescence intensity graphs along a straight line passing through the cell, and visualize the spatial fluorescence intensity distribution in 3D, Image J software was used.

Keywords: Microfluidic; CELLviewer; Spheroids; Imaging; Biomedical

\section{Introduction}

Cultivating cells and making cell models, in a biotechnological or biomedical laboratory, under controlled and favorable conditions, is essential for scientific research with aims in different fields of study: the use of stem cells in regenerative medicine, from the discovery of targets and cancer drugs, the production of therapeutic proteins and finally the modeling and characterization of diseases [1]. In vitro cell cultures can be isolated from normal or diseased tissues, can be grown as adherent monolayers or in suspension, and can be arranged to summarize some of their 
functions, in two or three dimensions [2,3].

The two-dimensional (2D) approach involves cells that proliferate on flat substrates, resulting in a monolayer cell far from the type of cells that grow in vivo. They are devoid of cellcell and cell-matrix interactions in the microenvironment [4].

The traditional 2D culture systems growth alone or in co-culture on plates, in which experiments supported by in vitro imaging are conducted for different functional, pharmacological, toxicological, and even clinical applications; they have long been widely used and already known for the nature of their cost and high repeatability. However, 2D culture systems cannot reach a stage of 3D organization equal to in vivo, due to the disadvantages associated with the lack of specific tissue architecture, mechanical-biochemical signals, cell-cells and Extracellular Matrix (ECM) [5-7].

One issue with conventional 2D cell culture systems is the inadequate quality and quantity of ECM, which is fundamental to the support of the structure by facilitating communication between the different cell populations embedded in the matrix by imparting mechanical properties to the tissues [8]. Cells in 2D culture are not surrounded by ECM and therefore are different from the structure of an in vivo cell system, as they cannot: migrate, polarize and differentiate in response to [9-12]. Despite their proven value in biomedical research, 2D models cannot support differentiated and cell-specific functions in tissues or accurately predict in vivo tissue functions and drug and biological modulator activities [1316]. These limitations have led to a growing interest in the development of more complex models, such as those that incorporate multiple cell types or involve cell modelling and in three-dimensional (3D) models, which better represent the spatial and chemical complexity of living tissues [17-19]. This lack has led to the development of three-dimensional (3D) cell culture models to improve in vitro research systems, more accurately recapitulating the in vivo state in which: cell morphology, interactions and specific architecture most represent that of native tissues [20].

Three-dimensional (3D) cell culture better replicates the physiological interactions between cells and their environment than traditional two-dimensional (2D) cell culture cells. Particular types of cell lines have the ability to self-aggregate into 3D aggregates called cell spheroids when grown in suspension [21].

The terms spheroid and organoid are commonly used when addressing the topic of 3D cell culture, but this terminology has been used interchangeably. There are several distinct differences between them for equal merit of definition, implementation and utility as an advanced model of scientific research $[20,22,23]$.

\section{D Models}

\section{Definitions and Current Terminology}

Spheroids and organoids both refer to 3D culture models that have specific organization and architecture, not achievable through 2D monolayer cell cultures. There is currently no universally well-defined nomenclature for these models and in the literature.

The term spheroid, first coined in the 1970s, was noted that some Chinese hamster lung cells when dissociated were capable of forming almost perfectly spherical suspended cell aggregates grown in rotating flasks [20]. Since that time, spheroids have been generated from many types of primary cells and cell lines in a multitude of methods such as hanging drop, low cell attachment plates, micropatterned surfaces or rotating bioreactors [3,20,24-26].

The term spheroid is currently used in research where cancer cells constitute the invaluable multicellular model of tumor spheroid (MCTS) for studying solid tumor biology [20].

The spheroid is used to describe tumor models in which the 3D structure has been formed from standard immortalized cell lines traditionally grown in 2D. Spheroid cultures can also be generated from primary tumor cells [27].

The MCTS spheroid is characterized by having a subdivision into regions. Starting from the most external there is the proliferation of cells, an internal zone in which quiescent cells reside and finally the central zone composed of a necrotic core $[20,24]$. This arrangement mimics the cellular heterogeneity observed in solid tumors [28].

The cell arrangement within the MCTS emulates cell morphology, proliferation, oxygenation, nutrient uptake, waste excretion, and drug uptake in vivo [20,29].

The embryoid bodies are 3D stem aggregates capable of giving rise to cells that form the 3 germ layers and in turn differentiate into aggregates [20]. When incorporated into Matrigel without the addition of specific growth factors, the aggregates form buds and further develop into discrete regions [30].

Embryoid bodies, mammospheres, hepatospheres and neurospheres are the most widely used spheroid models as a MCTS from cancer cells. They are used as models of avascular cancer to obtain information on cancer invasion and therapeutic cancer screening of metastases [20,30]. The subdivision of the population that makes up the spheroid heterogeneously, between those in proliferation and not, 
exposes the spheroid model to an oxygen gradient that emulates the physiochemical gradients found in solid tumors [28]. Some advantages of spheroidal 3D models are that they are relatively easy and quick to model, versatile depending on the study under consideration, and cheap enough to tackle high-throughput screening [31].

The organoid was previously defined as an aggregation of cells that contained differentiated cells with some tissuelike structures [32]. The description currently associated with the term organoid is that of a 3D structure grown from organ stem cells or progenitor cells composed of organspecific cell types that self-organize through cell selection and a space-limited lineage that mimics at least one organ function $[20,33,34]$.

Organoid describes 3D biological structures grown in vitro that resemble there in vivo counterpart in architecture and function [23]. Characteristics of organoids include self-organization, multicellularity, and functionality [20]. They then recap the microanatomy of the organs with differentiated cell types specific to each organ [35].

Organoids can be mainly classified into two macro categories: those deriving from adult tissues and those deriving from pluripotent stem cells [3,20,24,36,37]. Organoids contain multiple cell types organized into structures that resemble the organ of interest and exhibit some of the functions of the study target organ[20].In general, a higher order of self-assembly is found in organoids than in spheroidal cultures, which are more simplistic, with little or no tissue structure characteristics [23]. The formation of organoids depends more on the presence of biological or synthetic matrices [29]. The interaction between the cell and the ECM is essential for survival, proliferation, differentiation and migration [25]. The ECM also provides a physical structure on which cells can move and grow in 3D [38]. Spheroid cultures are less dependent on matrices for their formation, capable of forming in both scaffold-free and scaffold-based conditions [20].

\section{Advances in cell cultures}

The development of 3D culture is closely related to understanding the cellular microenvironment and the importance of the ECM as a regulator of morphogenesis and tissue function [39]. The ECM is made up of insoluble collagen proteins and fibers. Collagen provides rigid structures and physical support, while ECM proteins, including fibronectin, laminin and integrins, are involved in signaling processes [24]. The basement membranes, or lamina propria, provide epithelial and endothelial cells with structural and organizational stability, act as a barrier, and transduce signals and other functions [40].

Matrigel, isolated from chondrosarcoma in 1977, the first basement membrane extract, has been repeatedly used for 3D cell cultures and for suspension methods to form spheroids [41]. Following Matrigel and the characterization of ECM components, 3D culture approaches incorporate the use of matrices, animal-derived hydrogels or synthetic hydrogel networks with ECM components. Providing physical support and biochemical signals that recapitulate cellmatrix interactions. Thus incorporating biological aspects of morphogenesis, homeostasis and recapitulating spatial signals that appear to be absent in $2 \mathrm{D}$ cultures $[29,41]$.

An advantage of cell growth in 3D cultures is that they are reflected more accurately in vitro than cells growing in monolayer cultures. This is because cells do not normally grow or interact in isolation; rather, they form interactions with other cells and the microenvironment that surrounds them [42]. By affecting gene expression, the distribution of proteins such as receptors, transcription factors or cell cycle regulators [43].

Cell-cell interactions in 3D cultures involve cells of the same type, as can occur in spheroids grown from a tumor cell line. Communications can involve multiple cell types when co-cultured with other cells [20]. Cultures of tumor spheroids exhibit tumor-like cytoarchitecture and contain metabolic gradients of gas and nutrients. As the distance from the periphery increases, the availability of oxygen and glucose decreases, reflecting the state of hypoxia and nutrient deprivation, characteristic of neoplasms, which influence the reactivity and efficacy of the drug. Spheroid patterns also recapitulate lactate accumulation in tumors, which modulates tumor immunogenicity, reducing immune receptor expression and inhibiting effector cell action. These models also summarize the proliferative gradients of tumors: an outer layer of proliferating cells surrounds a layer of non-dividing but viable cells, defined as the drug-resistant dormant niche. The increase in cell death by apoptosis and necrosis leads to the accumulation of a necrotic core, which accumulates in the center of the spheroid $[25,33,44]$.

The biological features of 3D tumor models, such as modulation of immune receptor expression and accumulation of lactic acid, mimic the in vitro processes of tumor evasion from the immune system. They can therefore be used to evaluate immunotherapeutic approaches [29].

3D cultures recapitulate relevant physiological cellular processes, transforming into unique platforms for drug screening. 3D culture models replicate many factors affecting anticancer drug activity in vivo, proliferation 
gradients, cell-cell interactions and ECM-cell signaling, nonuniform exposure of cancer cells to oxygen and nutrients, microenvironment $[45,46]$.

Current strategies incorporate recent advances in stem cell biology, following ESC from mouse blastocysts in 1981 and humans in 1989, the introduction of somatic cell pluripotency factors generating iPSCs in 2006 [47].

The multitudes of advances in stem cell biology have enabled the formation of 3D organoid cultures [48]. Organoid culture technology has made huge strides in the last decade leading to the development of models, which mimic different organs including: intestine, stomach, pancreas, colon, liver and many more [20,36,49-52]. Organoids grown in 3D represent in vitro organ development, structural properties and organ functionality in vivo. They are used to model the development and physiology of organs in pathological states, and the fate of an organoid can be modulated through genetic editing, as occurs in genetic disorders and infectious diseases [20].

Organoids also have multiple applications in drug discovery and toxicity assays [53]. Disease modelling in organoids may involve pathogen infections or the targeted introduction of pathogenic mutations including inherited mutations or cancer development and progression [54].

Although the uses of 3D cultures recapitulate unique aspects of cell function, current 3D cultures represent reductionist models of the in vivo counterpart. Unfortunately, 3D models lack several aspects of organ biology and pathological processes such as the presence of surrounding tissue types, innervation, vascularity, the presence of immune cells and tumor stroma $[20,55,56]$.

\section{Co-culture system}

Co-culture systems have long been used to study the interactions between cell populations and are fundamental to cell-cell interaction studies. Such systems have become of particular interest to biologists for the study and design of complex multicellular 3D systems [57,58]. Co-culture allows a variety of cell types to be cultured together to examine the effect of one culture system on another, useful when examining the effect of one type of tissue, region, or how a particular molecule secreted leads to changes in development or physiology [57,59-61].

Co-culture is a cultivation set-up commonly adopted in 3D spheroid and organoid cultures, where two or more different cell populations are grown with some degree of contact with each other and the use of such a setup includes: study natural interactions between populations, improve crop success for certain populations or establish interactions between populations $[62,63]$.

2D co-culture is traditionally used to investigate the interactions between cancer cells and stromal cells in tumor tissues, but often fails to reproduce in vivo cell responses due to the highly artificial culture environment. Currently, 3D cell culture systems are being widely adopted in the fields of biological research, drug discovery and tissue regeneration $[63,64]$.

Compared to 2D monolayer cultures, cells in a 3D models culture, with or without a scaffold matrix, can exhibit more in vivo-like morphology and physiology such as proliferation rate and gene expression pattern [65]. The main advantages of 3D systems over 2D monolayers for co-culture studies include: 3D cultures enable the formation of spheroids or spatial cellular organization; Across the ECM and 3D cellular structure, gradients of nutrient, oxygen and growth factor can be created and play important roles in the tumor-stroma crosstalk; In 2D co-culture, different cell types are usually mixed and grow on the same monolayer. In contrast, 3D systems allow the co-cultured cells to be situated in different compartments or matrix layers [63].

3D cultures support co-cultivation and crosstalk of multiple cell types, which regulate development [66].

\section{D Models Become Fundamental for Analyzing Cellular Relationships}

Some 3D models provide great results in representing tissue structures in the physiological field compared to twodimensional 2D cell culture [10,19,67-69]. The fabrics have a hierarchical structure that contains micro-architecture features that can be studied on many length scales. These include the subcellular/cellular scale $(1-10 \mu \mathrm{m})$, which affects cellular function; the multicellular scale $(10-100$ $\mu \mathrm{m})$, which determines the type and degree of intercellular interactions; and the tissue scale $(100-1000 \mu \mathrm{m})$, which correspond $[7,70]$. Deciphering population heterogeneities is a long-standing goal in cellular biology. At the level of single cells, such heterogeneities are usually observed at the genomic, transcriptomic or phenotypic levels [71]. In general, spheroids, self-organizing and heterogeneous cell aggregates up to $400-500 \mu \mathrm{m}$ in size, are used for research, resulting from the suspension or adhesion on the single-cell jamb or co-culture of more than [72]. Spheroidal models have advantages derived from their geometry and the possibility of developing effects in co-culture and sustainability generally long-term, as they mimic optimal cell-cell and cell-ECM physiological interactions, reproducibility and the similarity in protein-gene expression profiles. The use of these models is not transferable to cell types, as 3D spheroids of these cells 
tend to disintegrate or take unpredictable forms [21]. To avoid unpredictable and not currently useful developments, various types of scaffolds are manufactured and applied tools that control the development and structuring of spheroids' uniform dimensions [22].

The realization of an organoid, pseudo-organ, or neoorgan has in common some processes present in the various stages of development and formation of a living organism. This includes differentiation, proliferation, polarization, adhesion and precisely controlled apoptosis that combined with self-organization and multi-cellular pattern leads to the development of the various districts [73]. The Organoids or Tissues Organs are an in vitro 3D cell cluster derived from stem cells or progenitors and/or donors that spatially organize themselves in a similar way to their counterpart in vivo [74]. In the structuring and organizations of culture systems and especially of co-culture systems cells must maintain an adequate phenotype compatible with the external cellular environment and the duration of this phenomenon must be particularly protracted over time. For adhesion-dependent cells, interactions with the surrounding ECM and neighboring cells define the shape and organization of cells. One of the most surprising differences observed when comparing cell cultures in 2D and 3D is the morphological dissimilarity and their evolution over time. Cells grown in a monolayer tend to flatten on the bottom of the plate dish by adhering and spreading on the horizontal plane without expanding into the vertical dimension. The consequence is that cells grown in 2D have a forced apex-basal polarity. This polarity is probably relevant for certain cell types such as epithelial cells, but it is unnatural for most cells especially those of cubic or multifaceted type. The mesenchyme, if incorporated into a 3D ECM, take on a starry morphology and polarize only by bottom-up during migration $[16,75]$.

To support and guarantee the functional maintenance of a 3D structure, one must consider the structures and dynamics of regulatory networks, increasingly studied with live-imaging microscopy [76].

However, commercially available technologies that can be used for current laboratory needs are limited, although there is a need to facilitate the acquisition of cellular kinetics with a high spatial and temporal resolution, to elevate visual performance and consequently that of experimentation [10,77-79].

\section{Microfluidic Live Imaging}

2D models in Petri dishes allow for collective cell simulation and behaviours related to disease modelling and understanding but the advent of laboratory and organ devices on a chip shows that information obtained from
2D cell cultures on plates differs significantly from results obtained in microfluidic environments as they reflect more biomimetic aspects [30].

2D culture imaging does not allow to fully appreciate the morphology of the cell population and the threedimensionality of the sample, one of the reasons could be the unappreciated evolutionary changes. The use of imaging is an essential requirement for the study of the structural and functional morphology of the neo-organ, of its positioning / polarization and of cell differentiation, allowing the in vitro modeling of even the most complex organs [31]. Combining live imaging with the ability to retrieve individual cells of interest remains a technical challenge [80-82]. Combining imaging with precise cell retrieval is of particular interest when studying highly dynamic or transient, asynchronous, or heterogeneous cell biological and developmental processes [80]. Technological advances have enabled a broad array of live-cell imaging approaches to investigate cellular processes, many of which require optically demanding imaging modalities to achieve the necessary resolution and sensitivity [83]. A new technology based on newly developed microfluidics and imaging techniques can enable the management and identification of the phenotype, the biological activities of the present populations of the present populations without destroying the 3D of an organoid or derived in culture or co-culture of progenitor organs and/ or donors who self-organize in space/time like the in vivo $[74,84,85]$. However complete lab-on-a-chip devices that can work with the automated procedure and allows seeing the behavioural cities of cells or their alterations to support microfluidic system, have not been prevented in the literature usage [67].

Currently from what can be found in the bibliography, we have found that commercially the only equipment available to perform some specific protocols is CELLviewer [86,87].

The CELLviewer is a newly conceived and developed multi-technology instrumentation, combining and synchronizing the work of different scientific disciplines in the field of management of both simple and complex 3D culture systems, allows maintaining in the most natural conditions possible the three-dimensional structure, following it over time through high-definition time-lapse microscopy.

\section{CELLviewer}

\section{What exactly is CELLviewer?}

CellDynamics, Bologna, Italy, developed the CELLviewer, a lab-on-a-chip for managing cells or neo-organs in the absence of adhesion (Figure 1). This multi-technological device consists of a hardware tool and disposable 
components, which include microfluidic chips for inserting the sample. Environmental monitoring, automatic change of cultural media, the ability to insert individual cells or neo-organs, and optical analysis in light field, darkfield, and fluorescence microscopy are just a few of the device's features. Furthermore, CELLviewer technology enables remote execution of custom protocols via programmable software and automated execution. The CELLviewer is a revolutionary tool for time-lapse monitoring and imaging of suspended 3D models in a dynamic medium, as well as keeping biological units alive and mimicking the environment more naturally than other devices. By producing extremely finely balanced micro-currents, the CELLviewer device holds 3D models in suspension, counteracting the gravitational collapse of cells. These micro-currents perform two additional functions in addition to raising the dropping sample for gravity: they enable the biological sample to be transferred into three dimensions in a regulated and custom manner, and they allow the administration of external stimuli such as medications, fluorescent probes, growth factors, and more to be changed. Individual live cells or pseudo-organs have evolved within a suspension, been observed, and then inserted in a disposable cartridge using high-resolution timelapse fluorescence imaging. A disposable cartridge prevents contamination between one study and the next, and the basic device is surrounded by a set of measures that enable you to preserve the environment for cellular life; The currentgenerating channels are compatible with both spheroids and single cells, and the method for changing the culture soil and administering drugs allows for an airtight, sterilized link.

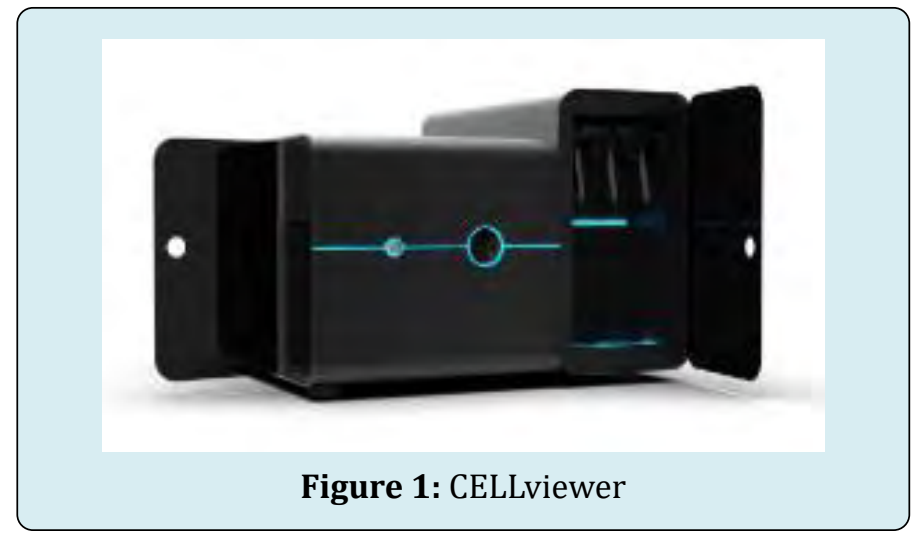

CELLviewer data can be used to observe and understand biological phenomena that occur naturally or are induced. The method contains many optical and electronic components to accomplish this, allowing individual cells or cell populations to be cultured and observed.

\section{Cartridge and Platform are two elements of the CELLviewer}

The key component of the device is a microfluidic integrated framework that incorporates various technologies to create a hybrid system that combines an incubator for 3D cell culture with a wide-field microscope for time-lapse, live imaging. The platform is made up of hardware and a physical interface that is inserted in a PC and works with a single-use proprietary cartridge Figure 2 . The cartridge is made up of multilayer plasma-bonded PDMS sheets that are crossed by a complex network of microfluidic channels of various diameters (ranging from $0.100 \mathrm{~mm}$ to $1 \mathrm{~mm}$ ) that cover a total volume of about $2 \mathrm{~mL}$. It's kept warm by an aluminum warming cover. Four hydrothermal pumps are used to create buffer microflows for sample management. CELLviewer's 13 plat form is a hybrid technology that combines a fluidic module, an optical device, electronics, and system management software. The fluidic module is designed for cartridge liquid management and is made up of motorized rotative valves, selection valves, and pinch valves. A motorized clamp attachment connects the cartridge to the hardware device.

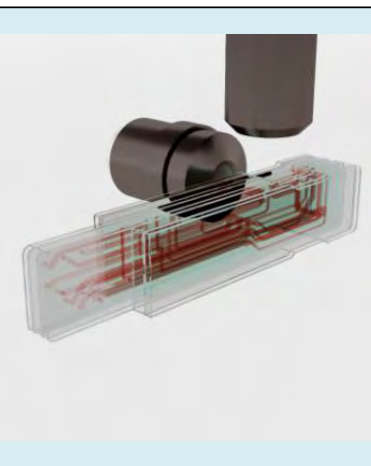

Figure 2: Cartridge

Three parallel research chambers with a rectangular form and a volume of 3 liters each make up the microfluidic circuit's central core. For sample loading and medium culture replacement with drug solution or fresh swab, each analysis chamber is located on the lower side of the microfluidic circuit. Four micrometric channels hit the top of each chamber to deliver buffer microflows that counteract gravity power, holding the sample suspended and in focus for time-lapse, live imaging.

Two cooperating light paths are used in the imaging setup to obtain the $x-y-z$ coordinates of a single floating sample in the study chamber. Vertical optics have a $2.5 \mathrm{X}$ magnification (NA $=0.08$ ) Olympus Life Science objective and an LD (low definition) camera, while time-lapse photography is done on a horizontal path with $20 \mathrm{X}(\mathrm{NA}=0.45)$ and $40 \mathrm{X}(\mathrm{NA}=0.60)$ Olympus Life Science objectives mounted on a motorized pistol for agile switching. The HD (high definition) camera (Hamamatsu Photonics) can capture up to 30 frames per second in full resolution of 4.0 megapixels and has a builtin cooling element, allowing for long-term acquisitions. With 
a white LED for sample positioning and bright-field imaging and 5 LED for multicolor Epi-fluorescence microscopy, illumination is based on an LED stroboscopic light source that reliably reduces sample photo damage. A 7-filter Pinkel penta-band package (Semrock Inc.) is included in the device, which is designed to image a sample simultaneously labeled with DAPI, FITC, TRITC, Cy5, and Cy7.

\section{What CELLviewer allows us to do}

Two experiments were carried out with different samples to validate the ability of CELLviewer to grow biological samples, but the viability of both was assessed using MitoGreen-dye, a fluorescence marker selectively permeable to live cells. Mitochondrial-targeted dyes may be used to determine cell status, such as apoptosis, high-energy, or mitochondrial membrane potential (MMP). MitoGreen is a fluorescent mitochondrial dye that has demonstrated green staining around the nuclear region inside the plasma membrane's edges, which conceptually overlaps the mitochondrial region, allowing researchers to study normal cell function in physiological states. MitoGreen-dye is a cellpermeable green fluorescent lipophilic dye that, when used at low concentrations, is selective for live cell mitochondria. Mitochondria play an important role in both cellular life and death. In addition to being the primary source of ATP, mitochondria often serve as a significant calcium buffer, regulating enzyme activity. Furthermore, ROS produced by mitochondrial electron transport chains can cause oxidative damage to cells. Because of these factors, staining mitochondria with fluorescent dyes, antibodies, or naturally fluorescent molecules aids in the analysis of their structure and role in both normal and pathophysiological states. The CELLviewer device allows for high-content time-lapse fluorescence imaging of live cells grown in suspension inside a disposable cartridge when drug solutions are dispensed to the sample chamber to visualize biological effects at the single cell stage. Following this application notes [88,89], single Jurkat cells and $M C F-7$ Spheroids are stained with MitoGreen, isolated in the disposable CELLviewer cartridge, and then time-lapsed in both the Bright-field and GFP channels. MitoGreen has a spectral similarity to FITC, which makes it excitable at $488 \mathrm{~nm}$. MitoTracker dyes may be used to determine the total mass of mitochondria or to investigate changes in mitochondrial mass as a result of various treatments. Commercial MitoTracker studies include

Cell Signaling's MitoTracker Green FM and ThermoFisher Scientific's MitoTracker. MitoTracker 15 Green dye stains the mitochondria of living cells without requiring MMP. MitoTracker Green is incompatible with fixation, and the signal can be obtained at wavelengths of 490 and $516 \mathrm{~nm}$ for excitation and emission, respectively [79].

\section{Experiment and Results}

\section{Assessment of single cell viability}

\section{$>$ Materials}

- Jurkat cells (ATCC)

- RPMI culture medium (Gibco, Life Technologies, Thermo Fisher Scientific)

- MitoGreen (PromoKine, PromoCell)

- CELLviewer imaging system

- CELLviewer 50 ml DOCK

\section{$>$ Methods}

The Jurkat cells were grown in RPMI 1640 soil supplemented with 2 mM L-glutamine, 10\% FBS, 100 units/ $\mathrm{mL}$ penicillin, and $100 \mathrm{mg} / \mathrm{mL}$ streptomycin at $37^{\circ} \mathrm{C}$ and $5 \% \mathrm{CO}_{2}$. Jurkat were washed and suspended in FBS culture soil at a final concentration of $5 \times 105$ cells $/ \mathrm{ml}$ before the experiments. The sample was then incubated in the dark at $37^{\circ} \mathrm{C}$ for 20 minutes with MitoGreen $200 \mathrm{mM}$. (PromoKine, PromoCell). The cells were centrifuged for 5 minutes at 2000 rpm to extract excess MitoGreen and resuspended in culture soil at 5\% to the CELLviewer work concentration of $5 \times 103$ cells $/ \mathrm{ml}$. After that, the sample is piped into a $50 \mathrm{ml}$ Falcon tube, which is then sealed with a $50 \mathrm{ml}$ CELLviewer DOCK. CELLviewer captures sample images in the Bright field and GFP channels at 0.5 fps with $20 \mathrm{X}$ magnification after isolation and fluid adaptive autofocus.

CELLviewer automatically captures time-lapse imaged sample for 4 hours in the GFP channel (Figure 3) and Brightfield channel (Figure 5) after single Jurkat cell isolation in the microfluidic cartridge and fluid adaptive autofocus.

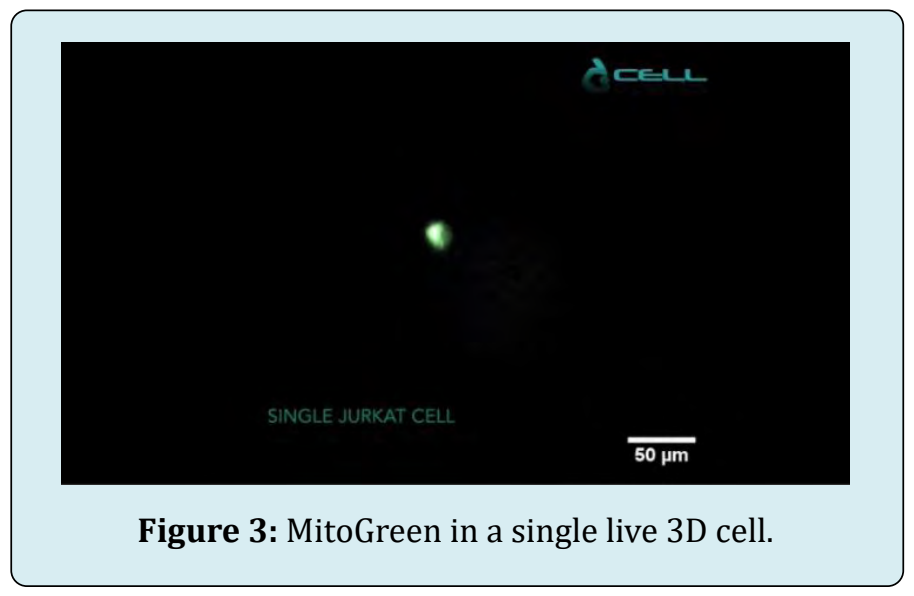

CELLviewer function concentration of $5 \times 103$ cells $/ \mathrm{ml}$ was achieved by resuspending MitoGreen in culture soil at $5 \%$. After that, the sample is piped into a $50 \mathrm{ml}$ Falcon tube, which is then sealed with a $50 \mathrm{ml}$ CELLviewer DOCK. Green staining was seen around the nuclear region and around the 
plasma membrane's edges. The scale bar is 50 meters.

The Measure function was used to determine the diameter of a single cell; the Plot profile plugin was used to generate fluorescence intensity graphics along a straight line that passes through the cell (Figure 4); and the 3D surface plot plug-in was used to show the distribution of spatial fluorescence intensity in 3D. MitoGreen is a fluorescent mitochondrial dye that showed green staining across the nuclear region and inside the edges of the plasma membrane, which conceptually overlaps the mitochondrial region, as shown in Figure 4 with the Plot profile and 3D surface plot, contributing to the study of normal cell function in physiological states.
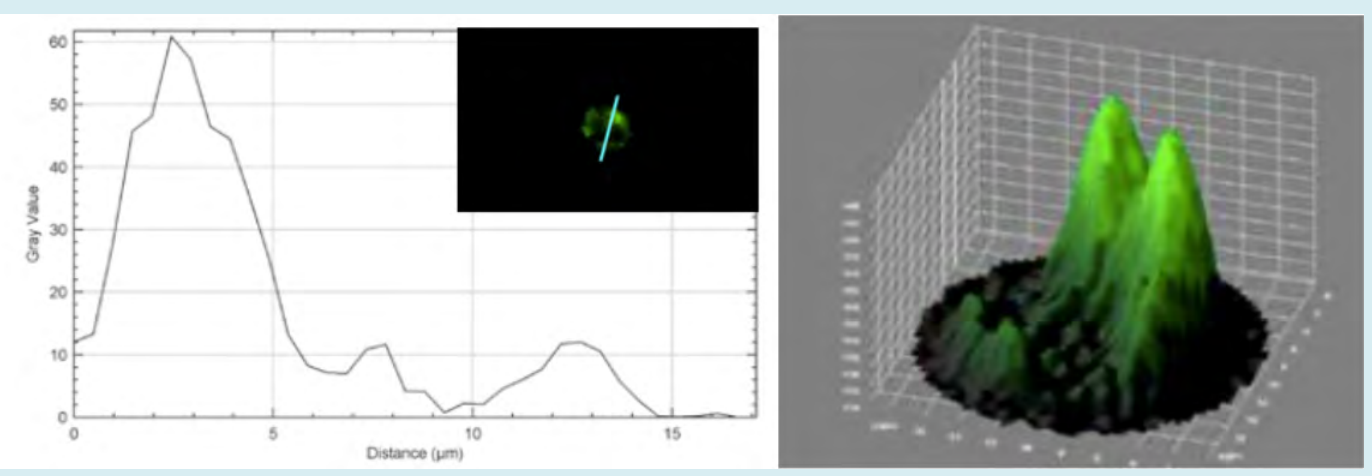

Figure 4: Image J software: Plot profile and 3D surface

Left: The plot profile plugin is used to generate fluorescence intensity graphics along a straight line that passes through the cell. Right: The 3D surface plot plug-in is used to visualize the distribution of spatial fluorescence intensity in 3D.

\section{The tracking of a single live cell}

In cancer line events, single-cell imaging is used to investigate cell heterogeneity. However, it has not been technically feasible to monitor the morphological changes of many individual initial cells over time $[90,91]$. With the CELLviewer method, single cell monitoring in 3D space can be combined with subsequent biochemical analyses of individually tracked cells, maintaining their identity traceable. Single-cell 2D monitoring allows for easier integration of subsequent biochemical studies and can be used as a stand-in for 3D measurements [74]. The 3D rotation of single cells in Figure 5 was successfully achieved experimentally on this basis.

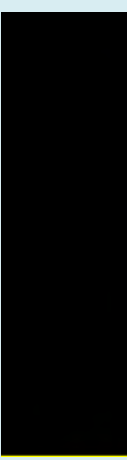

\section{SINGLE JURKAT CELL}

SIZE: 15 MICRON, PROBE: DIO

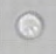

Figure 5: Single 3D live-cell.
As can be shown, when the fluid is flowed within the cartridge chamber, the individual cells are monitored by the optics. A single cell can retain a stable spatial location due to the effects of self-adaption and levitation. The scale bar is 50 meters.

\section{Generation of regular 3D spheroids for CELLviewer analysis}

\section{$>$ Materials}

- MCF-7 cell line (ATCC ${ }^{\circledR}$ HTB-22TM)

- DMEM with $1 \mathrm{~g} / \mathrm{L}$ glucose, sodium pyruvate and L-glutamine (Corning ${ }^{\circledR}$ Life Sciences)

- FBS 10\% (GibcoTM, Life Technologies, Thermo Fisher Scientific)

- L-glutamine $2 \mathrm{mM}$ (Sigma-Aldrich, Merck)

- Penicillin-Streptomycin solution (Sigma-Aldrich, Merck)

- Dulbecco's Phosphate Buffered Saline with $\mathrm{MgCl}_{2}$ and $\mathrm{CaCl}_{2}$ (Sigma-Aldrich, Merck)

- Percoll ${ }^{\circledR}$ solution, pH 8.5-9.5 $\left(25^{\circ} \mathrm{C}\right)$, cell culture tested (Sigma-Aldrich, Merck)

- Sphericalplate $5 \mathrm{D}^{\circledR}$ 24-well cell culture plate (Kugelmeiers AG)

- MitoGreen (PromoKine, PromoCell)

- CELLviewer imaging system and disposable cartridge

- CELLviewer 50 mL DOCK

- ImageJ software (US National Institutes of Health) 


\section{$>$ Methods}

MCF-7 cells were grown at $37{ }^{\circ} \mathrm{C}$ and $5 \% \mathrm{CO}_{2}$ in DMEM medium, supplemented with $2 \mathrm{mM}$ L-glutamine, 10\% FBS, 100 units/mL penicillin and $100 \mathrm{mg} / \mathrm{mL}$ streptomycin. Under a laminar flow sterile hood, the Sphericalplate 5D® plate is rinsed first with $1 \mathrm{~mL}$ of sterile PBS followed by 1 $\mathrm{mL}$ of complete medium. Every well is preloaded with 0,5 $\mathrm{mL}$ of complete medium, then $0.5 \mathrm{~mL}$ of cell suspension at a concentration of $3 \times 10^{5}$ cells / $\mathrm{mL}$ is pipetted in every well for a total volume of $1 \mathrm{~mL}$ per well. The plate is then incubated at $37^{\circ} \mathrm{C}$ and $5 \% \mathrm{CO}_{2}$ for 24 hours to promote spontaneous cell aggregation and uniform-sized 3D spheroids formation. The 3D Spheroids are withdrawn from 4 wells and trasferred in a $50 \mathrm{~mL}$ centrifuge tube. Sample is centrifuged for 5 minutes at $800 \mathrm{rpm}$ and the surnatant is discarded by gently aspirating. The pellet is resuspended in DMEM medium supplemented with Percoll ${ }^{\circledR}$, to improve sample stable focusing during long-term imaging in CELLviewer cartridge. Basicly, 3000 spheroids are resuspended in $20 \mathrm{~mL}$ of Percoll ${ }^{\circledR}$ supplemented DMEM medium to achieve a working concentration of 150 spheroids/mL. Consequently, MitoGreen 400nm solution is added to the culture medium and the sample is then pipetted inside a $50 \mathrm{~mL}$ Falcon tube closed with a CELLviewer $50 \mathrm{~mL}$
DOCK. MitoGreen probe uptake is thereby quantitatively analyzed within CELLviewer over the experiment lifetime. CELLviewer digitally isolates a single 3D spheroid and focuses the sample with a fluidic feedback mechanism. CELLviewer software, CELLcontrol, manages the imaging setup to automatically acquire sample images in Bright-field channel and GFP channel at 0,5 fps with 20X magnification. ImageJ software is used for image analysis using Measure function to calculate 3D spheroids diameter. All image acquisitions from FITC channel are stacked together and the same square shaped ROI (including spheroid borders) is applied to all the images. Adjust Brightness/Contrast function is used to homogeneously remove fluorescence background to all the stack images. Max Grey Value function is used to quantitatively assess fluorescence signal increase over the experiment lifetime.

\section{Result}

$1,5 \times 10^{5} \mathrm{MCF}-7$ cells are seeded in $1 \mathrm{~mL}$ of complete medium for every well to let them aggregate. Homogenoussized spheroid population shows mean diameter of $73 \pm 4$ $\mu \mathrm{m}$ at 24 hours of incubation and mean diameter of $73 \pm 5$ $\mu \mathrm{m}$ after 48 hours (Figure 6).

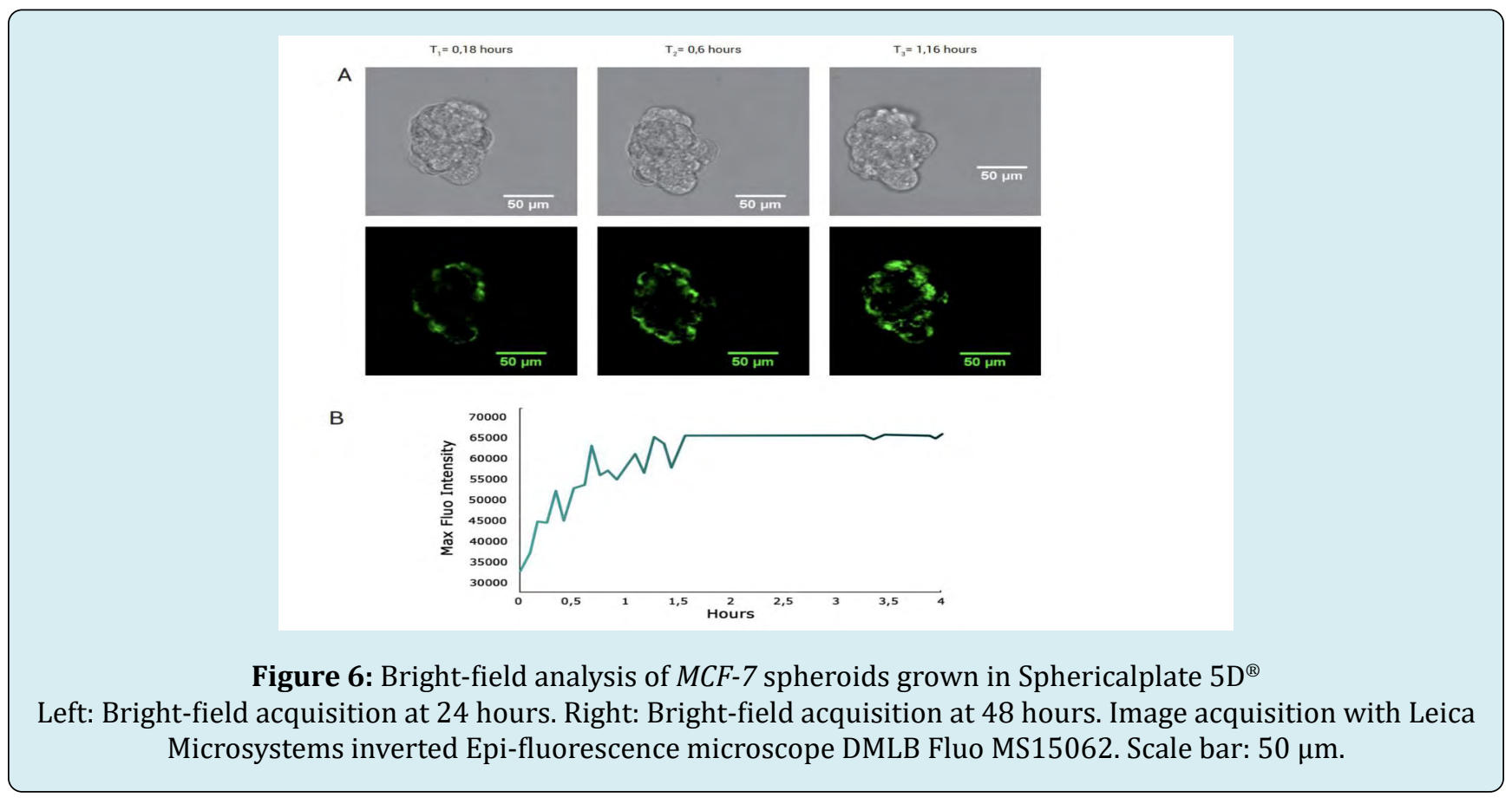

Spheroids diameter remains constant within 24 and 48 hours. Single 3D spheroids, composed of different cell lines (depending on customers' requirements) are digitally isolated in CELLviewer cartridge and a fluidic feedback mechanism focuses the sample in the analysis chamber for time-lapse, long-term culture and time-lapse live imaging. As shown in Figure 7 below, $100 \mu \mathrm{m}$ diameter MCF-7 spheroid is cultured for 4 hours in CELLviewer. A bright green fluorescent signal assesses spheroid viability, since MitoGreen conceptually overlaps mitochondrial regions of all viable cells composing 3D spheroids. Fluorescence signals gradually increases throughout the experiment time course, reaching maximum signal intensity within the first 1 hour and a half from the experiment start, due to MitoGreen probe 
progressive uptake by multicellular 3D spheroids.
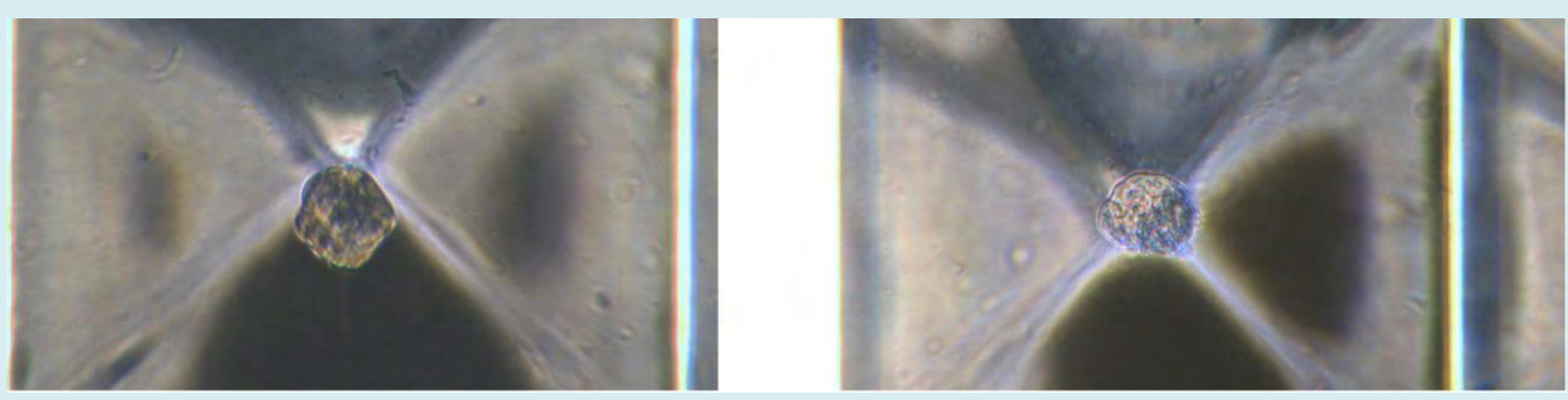

Figure 7: Analysis of MitoGreen labelled MCF-7 spheroid.

A: CELLviewer acquisitions in Bright-field channel (top) and in GFP-channel (below) at 3 different timepoints. B: Scatter Plot of MitoGreen Max Fluorescence Intensity (Grey Values) over the course of experiment. Scale bar: $50 \mu \mathrm{m}$.

\section{Discussion}

Compared to other devices in the literature $[67,72,77,84,92]$ the CELLviewer can perform experiments in real time in terms of physiology, toxicology, and clinical pharmacology, in addition to obtaining detailed images of current cellular morphology with resolution and high-quality data. Because of the software, the entire automated system allows for complete autonomy and protocol management, allowing the operator to focus on other tasks and increasing the project's productivity. A microfluidic device has been developed, and it has been shown that the 3D model can be spatially located. To direct the configuration, Epi-Fluorescence mitochondrial imaging was used. Furthermore, the evolution of cells and their 3D morphology can be acquired by using time-lapse imaging of cells. In conclusion, the proposed microfluidic technology can be used as a new platform solution to advance studies at the cellular level. The maximization of the times and ways of processing knowledge is a near and compulsory landing given the fundamental importance of the research economy. The preliminary experience with CELLviewer suggests that this equipment meets the needs of individual operators because it is made up of a synthesis of various integrated resources that can be controlled manually or automatically. This type of control system would make it easier to manage search dynamics remotely, using software and various devices, allowing the operator to focus on other tasks that involve his physical presence while maintaining the highest possible quality of the processes. Microfluidics' live-imaging of 3D models is an ideal solution for monitoring the optimum health of cells when bridging the difference between the 2D model and the 3D model [93]. With the advent of labon-a-chip technologies incorporating various laboratory operations into individual microfluidic networks and advancing the understanding of cell-cell and cell-biomaterial interactions, with the engineering of devices that can imitate the responses of cells, recent developments in the design, prototyping, and development of microfluidic systems have allowed new ways of approaching disease research. Since the multichannel structure provides cells with a 2D or 3D-like setting to current in vivo settings, these models are beginning to replace the most traditional cell culture systems, primarily Petri dishes.

Despite the progress made in the field of bioengineering over the last decade, particularly in terms of the success of these organ-on-chip systems as relevant research tools for the study of complex pathologies in a sustainable and cost-effective manner, there is still room for performance improvement. Understanding the behavior and interactions with the extracellular environment capable of mimicking in vivo success by advancing disease research is a longsought target and an ever-present research task in and of itself. Biomedical research on cellular systems of various complexities has progressed over time to the point that $2 \mathrm{D}$ is no longer sufficient. The fields of biotechnological and biomedical science are constantly searching for a method that recognizes as necessary, a model capable of reflecting the in vivo equivalent, with the goal of validating new methods with better outcomes in more physiological environments than the classic 2D monolayers. There are various and sometimes multiple causes that vary between $2 \mathrm{D}$ and 3D where the cell cycle of a single cell changes or the activity of cell populations differs in space-time within clusters, depending on the circumstances. The organization, configuration, and various structures are among the best-understood signals that the cell integrates to control a variety of main functions, including survival, differentiation, proliferation, migration, and polarization [94]. In order to research biology and cell function in vitro, the cells must be stripped of their native cellcell and multi-cell interactions and placed in a suspension 
environment with specific adhesion to the culture system. A deeper understanding of the three-dimensional model would aid in the further optimization of in vitro systems for the analysis of cell and tissue functions, as well as better translation to new therapeutics.

\section{Conclusion}

The key reason for using the co-culture method to perform experiments on 3D models is to better replicate the target in vivo, study the evolution of the model, and understand all types of cell-cell interactions, both natural and engineered. The extracellular environment has a significant effect on these parameters, which is sensitive to the experimental design's configuration. As a result, experimental systems capable of simulating the environment in which the 3D model will eventually be used are needed. The use of fluorescent dyes, antibodies, or fluorescent molecules to stain mitochondria will greatly aid research into their function and distribution, as well as cell viability in healthy and diseased people. The preliminary experience with CELLviewer suggests that this equipment responds to the needs of individual operators because it is made up of a synthesis of various integrated resources that can be controlled manually or automatically. A microfluidic device has been developed and demonstrated that the 3D model can locate the 3D model spatially, allowing experiments in physiology, toxicology, and clinical pharmacology to be carried out in real time. Because of the program, the whole automated system allows for complete autonomy and protocol management, allowing the operator to focus on other tasks and increasing the project's productivity. In conclusion, the proposed microfluidic technology can be used as a new platform solution to advance studies at the cellular level [95]. Fluorescence live-cell imaging is a vital method for researching the structure and dynamics of cells, as well as the internal and external components that make them up [96].

Given the first considerations, the CellDynamics Company is increasing the operating range of the CELLviewer machine, taking the cultivation of an even more complex 3D sample such as the zygote as a future approach. However, the main limitation of this machine turns out to be coupled with the complexity of that particular model: the speed of development and the maturation over time make it particularly difficult and unstable to manage with the system. These aspects need further investigation, but the gauntlet has been accepted.

\section{Acknowledgements}

This article and the research behind it would not have been possible without the support of my supervisor
Daniele Vigo. His need to train highly competent people, with the ability to grow independently, has been a source of inspiration since we had the first discussion outside from class. His subject matter in the Veterinary Biotechnology course has opened my eyes to a world so vast and rich in knowledge, fascinating and engaging. For the examination of him I began to do research and have never stopped since. The professor has always been a support within the university and he immediately became interested in me like no other university professor. He has always encouraged me to hurry up to get a degree and work together, unfortunately I failed in this and I'm sorry to have disappointed him. Until the end, at least until now he has always been lenient. Not only by sharing his future projects but also his complete willingness to help me in the next steps.

I thank Simone Pasqua and the members of CellDynamics who have been able to enhance their projects and create a truly incredible company, making possible what I have described in the previous chapters. Not only has this experience with them increased my vision but it has left me with a void that I hope to fill with what awaits me.

I also thank Gabriele Brecchia for his unfailing patience and for his kindness to grant a place in his office to a shaven person as I am. I am grateful to him for taking the time to listen to my fears and for introducing me to the world of European Congresses, which I would never have been able to access without him. Finally, I thank you for having endured the stench of burning when I welded the various components of the bioreactor.

Finally, I thank Giulio Curone for his wisdom shared with me, little pearls that have not been lost. A person who has long vision, it is no coincidence that you are Professor Vigo's favorite. I believe that without you it would not be the same. Like all the other people mentioned, I admire you for your commitment and wish you the best.

Finally, I thank my parents; I have no words for what I would like to write. Facts matter more than words and I want it to be so. Without them I would not have achieved anything, and their sacrifices will not be in vain, in the hope of being able to fulfil myself in life. A hug to everyone, thank you for your presence on this path.

\section{References}

1. Lukas Perkhofer, Pierre-Olivier Frappart, Martin Müller, Alexander Kleger (2018) Importance of organoids for personalized medicine. Pers Med 15(6): 461-465.

2. Boussommier-Calleja, Chapter 4.1 - In vitro models of cancer, in Bioengineering Innovative Solutions for 
Cancer (2020) S Ladamee J. Y. H. Chang, A c. di Academic Press. 273-325.

3. Claudia Corrò, Laura Novellasdemunt, Vivian SW Li (2020) A brief history of organoids. American Journal of Physiology-Cell Physiology 319(1): 151-165.

4. M Zurina, AI Shpichka, IN Saburina, NV Kosheleva, AA Gorkun, et al. (2018) 2D/3D buccal epithelial cell selfassembling as a tool for cell phenotype maintenance and fabrication of multilayered epithelial linings in vitro. Biomed Mater Bristol 13(5).

5. SA Hacking eA. Khademhosseini (2013) Cells and Surfaces in vitro, in Biomaterials Science: An Introduction to Materials: Third Edition, Elsevier Inc 408-427.

6. Saglam-Metiner P, Gulce-Iz S, Biray-Avci C (2019) Bioengineering-inspired three-dimensional culture systems: Organoids to create tumor microenvironment, Gene 686: 203-212.

7. Underhill GH, Galie P, Chen CS, Bhatia SN (2012) Bioengineering methods for analysis of cells in vitro. Annu Rev Cell Dev Biol 28: 385-410.

8. Bhattacharjee M, Coburn J, Centola M, Murab S, Barbero A, et al. (2015) Tissue engineering strategies to study cartilage development, degeneration and regeneration. Adv Drug Deliv Rev 84: 107-122.

9. Malta DFB, Reticker-Flynn NE, da Silva CL, Cabral JMS, Fleming HE, et al. (2016) Extracellular matrix microarrays to study inductive signaling for endoderm specification. Acta Biomater 34: 30-40.

10. Flaim CJ, Chien S, Bhatia SN (2005) An extracellular matrix microarray for probing cellular differentiation. Nat Methods 2(2): 119-125.

11. Gunawan F, Gentile A, Fukuda R, Tsedeke AT, JiménezAmilburu V, et al. (2019) Focal adhesions are essential to drive zebrafish heart valve morphogenesis. J Cell Biol 218(3): 1039-1054.

12. Li CY, Wood DK, Huang JH, Bhatia SN (2013) Flow-based pipeline for systematic modulation and analysis of 3D tumor microenvironments. Lab Chip 13(10): 1969-1978.

13. Choi J, Lich E, LeeJH (2016) Organogenesis of adult lung in a dish: Differentiation, disease and therapy. Dev Biol 420(2): 278-286.

14. Andrei L, Kasas S, Garrido IO, Stanković T, Korsnes MS, et al. (2020) Advanced technological tools to study multidrug resistance in cancer. Drug Resist Updat 48: 100658.
15. Kishida K, Pearce SC, Yu S, Gao N, Ferraris RP (2017) Nutrient sensing by absorptive and secretory progenies of small intestinal stem cells. Am J Physiol Gastrointest Liver Physiol 312(6): G592-G605.

16. Carter K, Lee HJ, Kyung-Sun N, Fernandes-Cunha GM, Blanco IJ, et al. (2019) Characterizing the impact of 2D and 3D culture conditions on the therapeutic effects of human mesenchymal stem cell secretome on corneal wound healing in vitro and ex vivo. Acta Biomater 99: 247-257.

17. Arciero JC, Mi Q, Branca MF, Hackam DJ, Swigon D (2011) Continuum model of collective cell migration in wound healing and colony expansion. Biophys J 100(3): 535543.

18. Allen JW, Bhatia SN (2002) Engineering liver therapies for the future. Tissue Eng 8(5): 725-737.

19. Allen JW, Hassanein T, Bhatia SN (2001) Advances in bioartificial liver devices. Hepatology 34(3): 447-455.

20. (2020) 3D Model Systems: Spheroids, Organoid and Tissue Model Systems. Cell Culture Dish.

21. Costa EC, Moreira AF, de Melo-Diogo D, Gaspar VM, Carvalho MP, et al. (2016) 3D tumor spheroids: an overview on the tools and techniques used for their analysis. Biotechnol Adv 34(8): 1427-1441.

22. (2019) A practical guide for precision cancer research using tumor-organoids. The PreCanMed technical Hanfbook.

23. Dong-Woo, et al. (2019) 3D Bioprinting. Springer New York LLC.

24. Organoid Culture Handbook.

25. Katt ME, Placone AL, Wong AD, Xu ZS, Searson PC (2016) In Vitro Tumor Models: Advantages, Disadvantages, Variables, and Selecting the Right Platform. Front Bioeng Biotechnol 4: 12.

26. M Capuzzo e D Vigo (2020) Updated modifications about Spinœ Bioreactor. J Eng Technol Appl Sci 5: 53-56.

27. Yoshida T, Sopko NA, Kates M, Liu X, Joice G, et al. (2019) Impact of spheroid culture on molecular and functional characteristics of bladder cancer cell lines. Oncol Lett 18(5): 4923-4929.

28. Johann DJ, Rodriguez-Canales J, Mukherjee S, Prieto DA, Hanson JC, et al. Approaching Solid Tumor Heterogeneity on a Cellular Basis by Tissue Proteomics Using Laser Capture Microdissection and Biological 
Mass Spectrometry. J Proteome Res 8(5): 2310-2318.

29. Boucherit N, Gorvel L, Olive D (2020) 3D Tumor Models and Their Use for the Testing of Immunotherapies. Front Immunol 11: 603640.

30. Zhou J, Zhang Y, Lin Q Liu Z, Wang H, et al. (2010) Embryoid bodies formation and differentiation from mouse embryonic stem cells in collagen/Matrigel scaffolds. J Genet Genomics 37(7): 451-460.

31. Langhans SA (2018) Three-Dimensional in Vitro Cell Culture Models in Drug Discovery and Drug Repositioning. Front Pharmacol 9: 6.

32. Simian M, Bissell MJ (2017) Organoids: A historical perspective of thinking in three dimensions. J Cell Biol 216(1): 31-40.

33. The Organoid Culture Handbook: R\&D Systems.

34. The Organoid Culture Handbook.

35. Yin X, Mead BE, Safaee H, Langer R, Karp JM, et al. (2016) Engineering Stem Cell Organoids. Cell Stem Cell 18(1): 25-38.

36. Bing-Ying X, Ai-Wen W (2016) Organoid Culture of Isolated Cells from Patient-derived Tissues with Colorectal Cancer. Chin Med J (Endl) 129(20): 24692475.

37. Balakrishnan S, Atal S, Ray A, Pravin CA, Nanda M (2020) Organoids: An invaluable tool in pharmacology. Indian J Pharmacol 52(5): 422-429.

38. Shah SB, Singh A (2017) Cellular self-assembly and biomaterials-based organoid models of development and diseases. Acta Biomater 53: 29-45.

39. Rodrigues T, Kundu B, Silva-Correia J, Kundu SC, Oliveira JM, et al. (2018) Emerging tumor spheroids technologies for 3D in vitro cancer modelling. Pharmacol Ther 184: 201-211.

40. Parsons' D, Skinner W (2001) A novel approach to examining and correlating function, structure \&amp; chemical-architecture in airway epithelium. 6(Suppl 1): A42.

41. Kleinman HK, Martin GR (2005) Matrigel: Basement membrane matrix with biological activity. Semin Cancer Biol 15(5): 378-386.

42. De Souza Moraes G, Wink MR, Klamt F, Silva AO, da Cruz Fernandes M (2020) Simplified low-cost methodology to establish, histologically process and analyze three- dimensional cancer cell spheroid arrays. Eur J Cell Biol 99(5): 151095.

43. Foglietta F, Canaparo R, Muccioli G, Terreno E, Serpe L (2020) Methodological aspects and pharmacological applications of three-dimensional cancer cell cultures and organoids. Life Sci 254(1): 117784.

44. Lee F, Iliescu C, Yu F, Yu H (2018) Constrained spheroids/ organoids in perfusion culture. Methods Cell Biol 146: 43-65.

45. 3D High-Content Screening of Organoids for Drug Discovery.

46. Li L, Zhou Q, Voss TC, Quick KL, LaBarbera DV (2016) High-throughput imaging: Focusing in on drug discovery in 3D. Methods 96: 97-102.

47. Takahashi $\mathrm{K}$, Yamanaka S (2006) Induction of pluripotent stem cells from mouse embryonic and adult fibroblast cultures by defined factors. Cell 126(4): 663-676.

48. Hynds RE, Giangreco A (2013) Concise review: the relevance of human stem cell-derived organoid models for epithelial translational medicine. Stem Cells 31(3): 417-422.

49. Saglam-Metiner P, Gulce-Iz S, Biray-Avci C (2019) Bioengineering-inspired three-dimensional culture systems: Organoids to create tumor microenvironment. Gene 686: 203-212.

50. Zanoni M, Cortesi M, Zamagni A, Arienti C, Pignatta S (2020) Modeling neoplastic disease with spheroids and organoids. J Hematol Oncol 13(1): 97.

51. Augustyniak J, Bertero A, Coccini T, Baderna D, Buzanska $\mathrm{L}$, et al. (2019) Organoids are promising tools for speciesspecific in vitro toxicological studies. J Appl Toxicol 39(12): 1610-1622.

52. Nath S, Devi GR (2016) Three-dimensional culture systems in cancer research: Focus on tumor spheroid model. Pharmacol Ther 163: 94-108.

53. Nunes AS, Barros AS, Costa EC, Moreira AF, Correia IJ (2019) 3D tumor spheroids as in vitro models to mimic in vivo human solid tumors resistance to therapeutic drugs. Biotechnol Bioeng 116(1): 206-226.

54. Engevik KA, Matthis AL, Montrose MH, Aihara E (2018) Organoids as a Model to Study Infectious Disease. Methods Mol Biol 1734: 71-81.

55. Jackson EL, Lu H (2016) Three-dimensional models for studying development and disease: moving on from 
organisms to organs-on-a-chip and organoids. Integr Biol(Camb) 8(6): 672-683.

56. Ishiguro $\mathrm{T}$, Ohata $\mathrm{H}$, Sato $\mathrm{A}$, Yamawaki K, Enomoto $\mathrm{T}$, et al. (2017) Tumor-derived spheroids: Relevance to cancer stem cells and clinical applications. Cancer Sci 108(3): 283-289.

57. Carter M, Shieh J (2015) Guide to Research Techniques in Neuroscience (Second Edn) Academic Press pp: 418.

58. Coculture-an overview | Science Direct Topics. https:// www.sciencedirect.com/topics/biochemistry-geneticsand-molecular-biology/coculture (consultato gen. 26, 2021).

59. Torre M L, Eleonora Munari, Elena Albani, Paolo E Levi Setti, Simona Villani, et al. (2006) In vitro maturation of human oocytes in a follicle-mimicking three-dimensional coculture. Fertil Steril 86(3): 572-576.

60. Three-dimensional hormone-free coculture for the maturation of swine oocytes : an in vitro fertilisation test. https://air.unimi.it/handle/2434/27556 (consultato gen. 26, 2021).

61. Faustini M, Curone G, Torre M L, Vigo D (2018) Bioencapsulation of Oocytes and Granulosa Cells., Methods Mol Biol 1817: 89-93.

62. Goers L, Freemont P, Polizzi KM (2014) Co-culture systems and technologies: taking synthetic biology to the next level. J R Soc Interface 11(96): 20140065.

63. Xin X, Yang H, Zhang F, Yang ST (2019) 3D cell coculture tumor model: A promising approach for future cancer drug discovery. Process Biochem 78: 148-160.

64. Haycock JW (2011) 3D Cell Culture: A Review of Current Approaches and Techniques. Methods Mol Biol pp: 1-15.

65. Li Y, Yang ST (2001) Effects of three-dimensional scaffolds on cell organization and tissue development , Biotechnol. Bioprocess Eng 6(5) pp: 311-325.

66. Sawant S, Harsh Dongre, Archana Kumari Singh, Shriya Joshi, Daniela Elena Costea, et al. (2016) Establishment of 3D Co-Culture Models from Different Stages of Human Tongue Tumorigenesis: Utility in Understanding Neoplastic Progression. PLOS ONE 11(8) pp: e0160615.

67. Bhatia SN, Ingber DE (2014) Microfluidic organs-onchips. Nat Biotechnol 32(8) pp: 760-772.

68. Ozbolat T (2016) 3D Bioprinting: Fundamentals, Principles and Applications. Elsevier Inc.
69. Reticker Flynn NE, David Braga malta F, Monte Winslow M, John Lamar M, Mary Xu J, et al. (2012) A combinatorial extracellular matrix platform identifies cell-extracellular matrix interactions that correlate with metastasis. Nat Commun (3).

70. Underhill GH, Khetani SR (2017) Bioengineered Liver Models for Drug Testing and Cell Differentiation Studies, Cell Mol Gastroenterol Hepatol 5(3): 426-439.

71. Anagnostidis V, Sherlock B, Metz J, Mair P, Hollfelder F, et al. (2020) Deep learning guided image-based droplet sorting for on-demand selection and analysis of single cells and 3D cell cultures. Lab Chip 20(5): 889-900.

72. Wechsler ME, Shevchuk M, Peppas NA (2020) Developing a Multidisciplinary Approach for Engineering Stem Cell Organoids. Ann Biomed Eng 48(7): 1895-1904.

73. Gline S, Kaplan N, Bernadskaya Y, Abdu Y, Christiaen L (2015) Surrounding tissues canalize motile cardiopharyngeal progenitors towards collective polarity and directed migration. Development 142(3): 544-554.

74. De Souza N, (2018) Organoids. Nat Methods 15(1):23.

75. Rubashkin MG, Ou G, Weaver VM (2014) Deconstructing signaling in three dimensions. Biochemistry 53(13): 2078-2090.

76. Rios C, Clevers H (2018) Imaging organoids: a bright future ahead. Nat Methods 15(1): 24-26.

77. Underhill GH, Galie P, Chen CS, Bhatia SN (2012) Bioengineering methods for analysis of cells in vitro. Annu Rev Cell Dev Biol 28: 385-410.

78. Bhatia SN, Underhill GH, Zaret KS, Fox IJ (2014)Cell and tissue engineering for liver disease. Sci. Transl. Med (6): 245.

79. Bhatia SN, (2006) Cell and tissue-based sensors. in Biosensing, Springer Netherlands pp: 55-65.

80. Mulas C, Andrew C Hodgson, Timo N Kohler, Chibeza C Agley, Peter Humphreys, et al. (2020) Microfluidic platform for 3D cell culture with live imaging and clone retrieval. Lab Chip 20(14): 2580-2591.

81. Luro S, Potvin Trottier L, Okumus B, Paulsson J (2020) Isolating live cells after high-throughput, long-term, time-lapse microscopy. Nat Methods 17(1): 93-100.

82. Zheng Y, Shao Y, Fu J (2021) A microfluidics-based stem cell model of early post-implantation human development. Nat Protoc 16(1): 309-326. 
83. Tepperman A, Zheng DJ, Taka MA, Vrieze A, Le Lam A, et al. (2020) Customizable live-cell imaging chambers for multimodal and multiplex fluorescence microscopy. Biochem Cell Biol 98(5): 612-623.

84. Albrecht DR, Underhill GH, Resnikoff J, Mendelson A, Bhatia SN, et al. (2010) Microfluidics-integrated timelapse imaging for analysis of cellular dynamics. Integr Biol 2(5-6): 278-287.

85. Fatehullah A, Tan SH, Barker N (2016) Organoids as an in vitro model of human development and disease. Nat Cell Biol 18(3): 246-254.

86. Capuzzo M, Vigo D, Brecchia G, Curone G, Pasqua S, et al. (2020) Microfluidic live-imaging with CELLviewer technology to perform biotechnological tasks. J Eng. Technol Appl Sci 5(6): 27-34.

87. < the CellViewer.pdf> .

88. AN_MKT_01 Epi-Fluorescence mitochondrial imaging in live single cells.pdf.

89. AN_MKT_02_Generation of regular 3D spheroids with Sphericalplate 5D for the subsequent CELLviewer analysis.pdf.

90. Ramasamy TS, Ong ALC, Cui W (2018) Impact of ThreeDimentional Culture Systems on Hepatic Differentiation of Puripotent Stem Cells and Beyond. Adv Exp Med Biol 1077 pp: 41-66.

91. Rijal G, Li W (2018) Native-mimicking in vitro microenvironment: An elusive and seductive future for tumor modeling and tissue engineering. J Biol Eng 12(1): 20 .

92. Wu Q, Pan Y, Wan H, Hu N, Wang P, et al. (2019) Research progress of organoids-on-chips in biomedical application. Chinese Sci Bull 64 (9): 902-910.

93. Robinson SK, Ramsden JJ, Warner J, Lackie PM, Roose T, et al. (2019) Correlative 3D Imaging and Microfluidic Modelling of Human Pulmonary Lymphatics using Immunohistochemistry and High-resolution $\mu \mathrm{CT}$. Sci Rep 9(1): 6415.

94. Huang L, Liang F, Feng Y (2019) A microfluidic chip for single-cell 3D rotation enabling self-adaptive spatial localization. J Appl Phys 126 (23): 234702.

95. Capuzzo A, Vigo D, Curone G, Gabriele Brecchia (2020) Automation in 3D cellular system in Live-Imaging with Microfluidic Technology CELLviewer®.

96. Araki S, Masayoshi Nakano, Mamiko Tsugane, Fumiko Sunaga, Mitsuru Hattori, et al. (2020) A simple microfluidic device for live-imaging of the vertical section of epithelial cells. Analyst 145(2): 667-674. 